\title{
Revisiting the Short-Wave Spectrum of the Sea Surface in the Light of the Weighted Curvature Approximation
}

\author{
Alexandra Bringer ${ }^{a, b}$, Bertrand Chapron $^{c}$, Alexis Mouche $^{d}$ and Charles-Antoine Guérin ${ }^{a, b}$ \\ a Université de Toulon (CNRS/INSU, IRD, MIO, UM 110), 83957 La Garde, France \\ ${ }^{b}$ Aix-Marseille Université (CNRS/INSU, IRD, MIO, UM 110) 13288 Marseille, France \\ ' IFREMER, Laboratoire d'Océanographie Spatiale, 29280 Plouzané, France \\ d CLS, Bât. le Ponant, Avenue La Pérouse, Technopôle Brest-Iroise 29280 Plouzané, France
}

\begin{abstract}
:
Existing models for the short-wave spectrum of the sea surface are not consistent with microwave satellite data when multi-bands and multi-incidence data sets are considered. We devise a simple parametric model for the short-wave omnidirectional spectrum of the sea surface on the basis of a three-band ( $\mathrm{C}, \mathrm{Ku}$, and $\mathrm{Ka}$ ) and multi-incidence (low, moderate, and large) data set and an improved analytical scattering model, namely the non-Gaussian Weighted Curvature Approximation. This spectrum is also constrained by several optical measurements which provide a priori conditions on the total and filtered mean-square slopes. It is compared with classical models such as Elfouhaily and Kudryavtsev unified curvature spectra. Significant differences are observed at wave numbers corresponding to the range of decimeter scales. The new spectrum is by construction fully consistent with the omnidirectional normalized radar cross section of the multi-band data set.
\end{abstract}

Keywords: Microwave ocean remote sensing ; omnidirectional spectrum 


\section{INTRODUCTION}

The characterization of the spectral properties of the sea surface has a long history dating back to the pioneering works of Phillips [1] and Pierson-Moskowitz ([2]). Today it is commonly accepted that the description of long waves has reached a mature state. The description of short gravity and capillary-gravity waves, however, is still undergoing research. Some recent improvements of the wave number spectrum in the millimeter and centimeter range of scales have been proposed in the last decade by different means. Among modern models, we can distinguish two paradigmatic spectra corresponding to conceptually different approaches. The first one is the popular model by Elfouhaily et al. [3], which unifies previous tank, in situ and optical measurements. The second is the model by Kudryavtsev et al. [4], which relies on a physical description of the the energy balance equation. A brief description of these models (henceforth referred to by the name of their first author, that is Elfouhaily and Kudryavtsev spectra) and their respective properties can be found in [5]. However, some differences if not contradictions are found between these models so that the question of the relevance and correctness of the short-wave curvature spectrum is is still open. Now, a precise description of the latter is more and more needed for an correct interpretation of the backscattered electromagnetic signal from the sea surface in the microwave regime. The aim of this article is to develop a spectral model which is fully consistent with a variety of scattering data at various incidences and frequency bands.

On the other hand any simulation or interpretation of sea surface backscatter is bound to a scattering model, which should be able to cover a large variety of sea state conditions and account for the relevant geophysical parameters. In this respect the simplest model is Bragg scattering as the resulting backscatter is merely proportional to the wavenumber spectrum. This property was used three decades ago in the pioneering work

Alexandra Bringer and Charles-Antoine Guérin are with the Université de Toulon (CNRS/INSU, IRD, MIO, UM 110), 83957 La Garde, France and the Aix-Marseille Université (CNRS/INSU, IRD, MIO, UM 110) 13288 Marseille. Bertrand Chapron is with IFREMER, Laboratoire d'Océanographie Spatiale, 29280 Plouzané, France. Alexis Mouche is with CLS, Bât. le Ponant, Avenue La Pérouse, Technopôle Brest-Iroise 29280 Plouzané, France. by Lawner and Moore [6] to estimate the short-wave wavenumber spectrum from the Marsen radar experiment. However, the Bragg regime is in general not attained at microwave frequencies and might appear as a very crude approximation. Now many progresses have been made recently in scattering models from the sea surface (e.g. [7]) with the construction of socalled unified models which can operate a dynamical transition between the different asymptotic regimes (Bragg diffraction as well as Physical and Geometrical Optics). However, an additional key ingredient in the scattering models is the inclusion of non-Gaussian characteristics of the sea surface. In the last two decade, a certain number of theoretical attempts have been made to incorporate non-Gaussian features in analytical models ([8], [9], [10], [11], [12], [13], [14], [15], [16], [17]). We will pursue along these lines with a recent, versatile and accurate electromagnetic model, namely the Weighted Curvature Approximation [18].

This study is concerned with the omnidirectional spectrum only, directional aspects being left for further research. As we will see, this will imply neglecting the influence of the two-point skewness function of the sea surface and rather consider the twopoint kurtosis function, which is related to kurtosis of slopes. The omnidirectional spectrum will be reconstructed from the Normalized Radar Cross Section (NRCS) arising from a set of multi-incidence and multi-frequency spaceborne and airborne data. The sole vertical polarization will be used for the inversion since horizontal polarization is in our opinion not sufficiently well described by the current models. Another limitation is the restriction of the geophysical parameters to the sole wind speed, additional descriptors of the sea state (e.g. swell, fetch, breaking, etc) being not taken in to account. Hence our final result should be thought as a mean spectrum over the variety of sea states at a given wind speed.

An useful guideline for the construction of the omnidirectional spectrum is an ensemble of integral constraints prescribed by various optical measurements, as recalled in section II. The scattering model is provided in section III. The reference data sets are presented in section IV. They are used to evaluate the performances of the existing classical spectra in term of simulation of the NRCS (section V). The new parametric model is introduced and discussed in section VI.

\section{The Integral CONStraints}

We recall that the omnidirectional wavenumber spectrum $S(k)$ is obtained by azimuthal integration of the directional spectrum $S(k, \phi)$ :

$$
S(k)=\int_{0}^{2 \pi} S(k, \phi) k d \phi
$$

The first few moments of the omnidirectional wavenumber spectrum $S(k)$ can be interpreted in terms of experimentally measurable quantities. This imposes some a priori global constraints on this unknown function and helps to reduce the domain of investigation. The integral of the wavenumber spectrum is proportional to the square of the significant wave height,

$$
H_{s}^{2}=16 \int_{0}^{\infty} d k S(k)
$$

The significant height is the most fundamental and intuitive parameter to describe the sea state. It can be easily measured by the time variations of a wave probe at a given location and is well quantified in terms of wind speed, fetch and swell. As the wavenumber spectrum loosely follows a $k^{-3.5}$ or $k^{-4}$ powerlaw decay, the value of the integral II.2 is imposed by the long 
waves. The second moment yields the so-called mean square slope (mss),

$$
\mathrm{mss}=\int_{0}^{\infty} d k k^{2} S(k)
$$

to which the small and large waves have comparable contributions. The mss is an important variable for the description of air-sea interactions as it controls the effective surface of exchange between the two media. Also, it describes the steepness of waves and is related to the breaking rate. The mss is also a key parameter in scattering of electromagnetic waves from the sea surface. In the Geometrical Optics limit of very short radiations it monitors the width of the scattering diagram. In the classical ([19]) or improved ([20]) Two-Scale Model it is involved in the tilt-modulation by large waves.

The mss can be accurately measured by optical means using either light boxes in laboratory $([21])$ or the sun glint in in situ conditions as was done in the celebrated experiment by Cox and Munk ([22]) henceforth referred to as CM. In the latter case a simple parametrization of the mss was obtained as a function of wind,

$$
\mathrm{mss}=0.003+5.1210^{-3} U_{12.5} \pm 0.004,
$$

where $U_{12.5}$ is the wind speed recorded at $12.5 \mathrm{~m}$ above the sea level. Note that the usual reference wind is taken at $10 \mathrm{~m}$ above the sea surface $\left(U_{10}\right)$, a convention which will be used throughout this paper. The CM mss corresponding to a given wind speed will be calculated by converting $U_{10}$ to $U_{12.5}$ using a classical von Karman logarithmic profile. In some instances one can also measure the partial or filtered mss which is the integration of the slope spectrum $k^{2} S(k)$ over a restricted range of scales,

$$
\Delta \mathrm{mss}=\int_{k_{1}}^{k_{2}} d k k^{2} S(k),
$$

This is the case for the "slick mss" observed by CM over artificially smoothed surfaces. In presence of oil slick it was estimated that wavelengths smaller than about $30 \mathrm{~cm}$ are damped due to the increased viscosity of the surface while longer waves are not modified. This implies a filtered mss (II.5) with cutoff values $k_{1}=0, k_{2} \simeq 2 \pi / 0.3$. The experimental values were parametrized in the same manner:

$$
\operatorname{mss}_{\text {slick }}=0.008+1.5610^{-3} U_{12.5} \pm 0.004
$$

The "slick" mss was later reevaluated by [23] by integration of a $k^{-3}$ spectrum up to the corresponding cutoff $\left(k_{s}=2 \pi / 0.3\right)$. A logarithmic rather than linear dependence was found,

$$
\operatorname{mss}_{\text {slick }}=4.610^{-3} \log \left(k_{s} / k_{0}\right),
$$

with $k_{0}=g / U_{10}^{2}$. For waves longer than $30 \mathrm{~cm}$ we will rely on this last relation. Another partial mss is provided by the airborne measurements described in [24]. In this experiment three vertical lasers set up in a triangle with one meter spacing are used to measure the slopes of one-meter chords in two directions at the sea surface. These segments are interpreted as the slopes of the large-scale process with wavelengths larger than about 1 meter and therefore give access to a low-pass filtered mss with $k_{1}=0, k_{2}=2 \pi$.

The fourth moment of the directional spectrum is the mean square curvature (msc), which describes the sharpness of waves. It is dominated by small waves and very sensitive to the shape of the wavenumber in the millimeter range. Hence it is a very discriminating parameter for the determination of the shortwaves wavenumber spectrum. It has also a strong effect on the

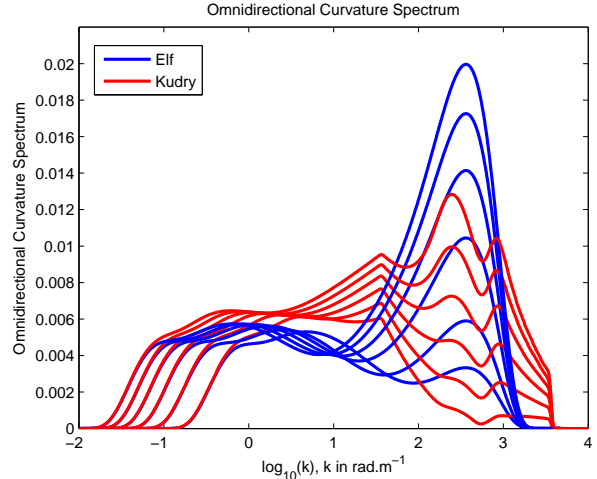

Fig. 1. Omnidirectional curvature spectrum $k^{3} S(k)$ after Elfouhaily and Kudryavstev model, binned by $2 \mathrm{~m} / \mathrm{s}$ wind speed starting at $5 \mathrm{~m} / \mathrm{s}$.

radiative properties of the surface, as the level of curvature determines the polarization ratio [15], [18]. There is, however, little knowledge of this parameter even though some recent works have provided its estimation using spaceborne radar data and a heuristic approach [25].

When integrated, the omnidirectional Elfouhaily and Kudryavtsev spectra give a consistent evaluation of the mss as compared with the reference value of $\mathrm{CM}$, with a maximum discrepancy of the order of 10-15\%. The msc, however, varies dramatically from one model to another. For instance, the total $\mathrm{msc}$ at $10 \mathrm{~m} / \mathrm{s}$ wind speed after Kudryavtsev spectrum is found more than three times larger than the same quantity after Elfouhaily model $\left(\simeq 20000 \mathrm{~m}^{-2}\right.$ versus $\left.6000 \mathrm{~m}^{-2}\right)$. This is due to important differences in the short wave curvature spectrum as shown on figure 1 .

\section{SCATTERING MODEL}

\section{A. The Weighted Curvature Approximation}

The calculation of the NRCS from the sea surface requires a so-called unified scattering model (e.g. [7]), that is a model which is able to cope simultaneously with large scales as well as small ripples. Such a model should be consistent with the asymptotic regimes in the appropriate limits. It should coincide with the Kirchhoff Approximation (KA) and Geometrical Optics for large Rayleigh parameter and Bragg theory for small Rayleigh parameter.

Since the historical Two-Scale model, there have been several attempts to build unified models resulting in more or less complicated formulations. Without being exhaustive we can mention the second-order small-slope approximation SSA2 [26], the Three-scale model [27], the local curvature approximation [28], the resonant curvature approximation [29], the cut-off invariant Two-Scale model [20] and the Weighted Curvature Approximation [28], [30]. This last model was recently revisited and simplified [18] in the case of the oceanic surface, where it was shown to enjoy an elementary formulation in terms of a combination of Bragg and Kirchhoff kernels. The simplicity and numerical efficiency of this model designates it as an excellent candidate for the inversion of remote sensing data, wherein a large number of direct problems have to be solved. The main limitation of WCA with respect to a more complex model such as SSA2 is its inability to predict correctly the cross-polarized components of the backscattering cross-section. In this context, however, this is not restrictive as only co-polarized components (namely the vertical polarization) will be used. We recall hereafter the geometry of the scattering problem. We consider a 
rough interface $z=\eta(\boldsymbol{r})$ separating air (upper medium) from water (lower medium). We denote $\boldsymbol{r}=(x, y)$ the coordinate in the horizontal mean plane. The surface is illuminated from above by an incident monochromatic, linearly polarized, plane wave with wave vector $\boldsymbol{K}_{\mathbf{0}}$. In backscattering configuration it is convenient to introduce the Ewald vector $\boldsymbol{Q}=-2 \boldsymbol{K}_{\mathbf{0}}$ together with its horizontal and vertical projections $\boldsymbol{Q}_{\boldsymbol{H}}$ and $Q_{z}$, respectively. In the Weighted Curvature Approximation (WCA) the scattering amplitude $(S)$ is sought as a correction of either the Kirchhoff or Bragg kernel:

$$
\begin{aligned}
S & =\frac{1}{Q_{z}} \int \frac{d \boldsymbol{r}}{(2 \pi)^{2}}\left(\mathbb{K}+\frac{i}{Q_{z}} \mathbb{T}(\boldsymbol{\nabla})[\eta]\right) \mathrm{e}^{-i \boldsymbol{Q}_{\boldsymbol{H}} \boldsymbol{r}} \mathrm{e}^{-i Q_{z} \eta} \\
& =\frac{1}{Q_{z}} \int \frac{d \boldsymbol{r}}{(2 \pi)^{2}}(\mathbb{B}-\mathbb{T}(\boldsymbol{\nabla} \eta)) \mathrm{e}^{-i \boldsymbol{Q}_{\boldsymbol{H}} \boldsymbol{r}} \mathrm{e}^{-i Q_{z} \eta}
\end{aligned}
$$

Again we refer to e.g. [7] for a rigorous definition of the scattering amplitude and the NRCS. The kernels $\mathbb{K}$ and $\mathbb{B}$ are the socalled Kirchhoff and Bragg tensors, respectively, which depend only on the scattering angles and the complex permittivity. The tensor $\mathbb{T}$ is quadratic in its argument. When formally applied to the gradient operator (i.e $\mathbb{T}(\boldsymbol{\nabla})[\eta]$ ), it yields a tensor-valued second-order derivative of roughness which is interpreted as a curvature correction of the mere tangent plane approximation. When applied to the surface gradient itself (i.e $\mathbb{T}(\boldsymbol{\nabla} \eta)$ ), it is a quadratic function of slopes. Contrarily to $\mathbb{K}$, the tensor $\mathbb{T}$ is polarization-dependent and thus responsible for the difference between horizontally and vertically polarized backscatter. The consistency between the alternative expressions (III.8) starting from the Bragg or the Kirchhoff regime is achieved with a mere integration by part together with an additional constraint on the kernel $\mathbb{T}$ :

$$
\mathbb{T}\left(\nabla \eta=-\boldsymbol{Q}_{H} / Q_{z}\right)=\mathbb{B}-\mathbb{K}
$$

These constraints are not sufficient to determine the kernel $\mathbb{T}$ uniquely. However, for linear sea surfaces the NRCS resulting from the different choices of the kernel was shown to follow closely a unique and simple expression independent on the kernel. To obtain a working formula at all angles of incidences and for non-linear surfaces, we therefore decided to choose the simplest admissible kernel which is proportional to the Laplacian operator $\left(\Delta \eta=\partial_{x x} \eta(x, y)+\partial_{y y} \eta(x, y)\right.$, where $\partial_{x x} \eta$ and $\partial_{y y} \eta$ are second-order partial derivatives),

$$
\mathbb{T}(\boldsymbol{\nabla})[\eta]=\mathbb{T}_{0} \Delta \eta,
$$

where $\mathbb{T}_{0}$ is a constant tensor:

$$
\mathbb{T}_{0}=(\mathbb{B}-\mathbb{K}) \frac{Q_{z}^{2}}{Q_{H}^{2}} .
$$

We recall that in the original approach $([28])$ the WCA kernel is defined as the difference $(\mathbb{B}-\mathbb{K})$ expressed in the variable $\left(\boldsymbol{Q}_{H}, Q_{z}\right)$ and with the formal replacement $\boldsymbol{Q}_{H} \rightarrow-Q_{z} \boldsymbol{\nabla} \eta$. This approach was shown ([18], section 3) equivalent to the simpler formulation. Under the assumption of a spatially homogeneous random sea surface, the global expression of the monostatic NRCS can then be written as a sum of three terms :

$$
\sigma^{W C A}=\sigma^{K A}+\sigma^{(12)}+\sigma^{(22)},
$$

where

$$
\sigma^{K A}=\frac{|\mathbb{K}|^{2}}{Q_{z}^{2}} \int \frac{d \boldsymbol{r}}{\pi} \mathrm{e}^{-\mathrm{i} \boldsymbol{Q}_{\boldsymbol{H}} \cdot \boldsymbol{r}}\left\langle\mathrm{e}^{\mathrm{i} Q_{\mathbf{Z}}(\eta(\boldsymbol{r})-\eta(\mathbf{0}))}\right\rangle
$$

is the classical expression of the NRCS in the KA and

$$
\begin{aligned}
\sigma^{(12)} & =2 \Re\left[\frac{\mathbb{K}^{*} \mathbb{T}_{0}}{Q_{z}^{2}} \int \frac{d \boldsymbol{r}}{\pi} \mathrm{e}^{-\mathrm{i} \boldsymbol{Q}_{\boldsymbol{H}} \cdot \boldsymbol{r}}\left\langle\Delta \eta(\boldsymbol{r}) \mathrm{e}^{\mathrm{i} Q_{\mathbf{z}}(\eta(\boldsymbol{r})-\eta(\mathbf{0}))}\right\rangle\right] \\
\sigma^{(22)} & =\left|\frac{\mathbb{T}_{0}}{Q_{z}^{2}}\right|^{2} \int \frac{d \boldsymbol{r}}{\pi} \mathrm{e}^{-\mathrm{i} \boldsymbol{Q}_{\boldsymbol{H}} \cdot \boldsymbol{r}}\left\langle\Delta \eta(\boldsymbol{r}) \Delta \eta(\mathbf{0}) \mathrm{e}^{\mathrm{i} Q_{\mathbf{z}}(\eta(\boldsymbol{r})-\eta(\mathbf{0}))}\right\rangle
\end{aligned}
$$

are polarization-dependent corrective terms. Here the brackets denote the ensemble average of the random process $\eta$. As usual the contribution of the coherent field in these expressions has been neglected in view of the elevated value of the Rayleigh parameter $Q_{z}\left\langle\eta^{2}\right\rangle$ in the bands of interest.

\section{B. WCA for linear surfaces}

When the sea surface is described in terms of a superposition of harmonics with random independent phases, the resulting random process is Gaussian in view of the central limit theorem. It is then entirely characterized by its autocorrelation function $(\rho)$ or, equivalently, its structure function $\left(S_{2}\right)$ :

$$
\rho(\boldsymbol{r})=\left\langle\eta \eta_{0}\right\rangle-\langle\eta\rangle^{2}, S_{2}(\boldsymbol{r})=2\left(\rho_{0}-\rho\right),
$$

where the notation $\eta, \eta_{0}$ or $\rho, \rho_{0}$ refers to a quantity evaluated at an arbitrary position $\boldsymbol{r}$ and at the origin, respectively. Here and everywhere, the position dependence in the different functions is omitted for simplicity and will remain implicit.

The different correlators involved in the NRCS can then easily expressed in terms of these latter functions:

$$
\begin{aligned}
\sigma^{K A} & =\frac{|\mathbb{K}|^{2}}{Q_{z}^{2}} \int \frac{d \boldsymbol{r}}{\pi} \mathrm{e}^{-\mathrm{i} \boldsymbol{Q}_{\boldsymbol{H}} \cdot \boldsymbol{r}} \mathrm{e}^{-Q_{z}^{2} S_{2} / 2}, \\
\sigma^{(12)} & =2 \Re\left[\frac{\mathbb{K}^{*} \mathbb{T}}{Q_{z}^{2}} \int \frac{d \boldsymbol{r}}{\pi} \mathrm{e}^{-\mathrm{i} \boldsymbol{Q}_{\boldsymbol{H}} \cdot \boldsymbol{r}} \mathrm{e}^{-Q_{z}^{2} S_{2} / 2}(\Delta \rho+\mathrm{mss})\right], \\
\sigma^{(22)} & =\frac{|\mathbb{T}|^{2}}{Q_{z}^{2}} \int \frac{d \boldsymbol{r}}{\pi} \mathrm{e}^{-\mathrm{i} \boldsymbol{Q}_{\boldsymbol{H}} \cdot \boldsymbol{r}} \mathrm{e}^{-Q_{z}^{2} S_{2} / 2}\left(\Delta^{2} \rho-Q_{z}^{2}|\Delta \rho+\mathrm{mss}|^{2}\right),
\end{aligned}
$$

where mss $=-(\Delta \rho)_{0}$ is the total mss of the surface. The omnidirectional version of a given quantity is defined as the angularaveraged value of this quantity over all possible azimuthal directions $\boldsymbol{Q}_{H}$. In an excellent approximation it can be shown and checked numerically that the omnidirectional NRCS is given by the same formula (III.16) applied to the omnidirectional correlation function. The corresponding integrals can then be efficiently performed using a preliminary radial integration using Bessel functions:

$$
\begin{aligned}
& \sigma_{\mathrm{omni}}^{K A}=2 \frac{|\mathbb{K}|^{2}}{Q_{z}^{2}} \int_{0}^{\infty} d r J_{0}\left(Q_{H} r\right) \mathrm{e}^{-Q_{z}^{2} S_{2} / 2} \\
& \sigma_{\mathrm{omni}}^{(12)}=4 \Re\left[\frac{\mathbb{K}^{*} \mathbb{T}}{Q_{z}^{2}} \int_{0}^{\infty} d r J_{0}\left(Q_{H} r\right) \mathrm{e}^{-Q_{z}^{2} S_{2} / 2}(\Delta \rho+\mathrm{mss})\right] \\
& \sigma_{\mathrm{omni}}^{(22)}=2 \frac{|\mathbb{T}|^{2}}{Q_{z}^{2}} \int_{0}^{\infty} d r J_{0}\left(Q_{H} r\right) \mathrm{e}^{-Q_{z}^{2} S_{2} / 2}\left(\Delta^{2} \rho-Q_{z}^{2}|\Delta \rho+\mathrm{mss}|^{2}\right),
\end{aligned}
$$

\section{WCA for nonlinear surfaces}

The hydrodynamic nonlinearities of the sea surface are known to have a significant impact on the scattering data. The socalled skewness function,

$$
S_{3}=\left\langle\left(\eta-\eta_{0}\right)^{3}\right\rangle,
$$


is known to be responsible for the upwind/downwind asymmetry in the classical scattering models [31], [15]. The so-called kurtosis function

$$
S_{4}=\left\langle\left(\eta-\eta_{0}\right)^{4}\right\rangle-3\left\langle\left(\eta-\eta_{0}\right)^{2}\right\rangle^{2}
$$

significantly impacts the shape of the backscattering diagram at small angles ([5]). The introduction of these non-Gaussian features in the statistical formulation of WCA is, however, far from being obvious and must be first established. This requires the calculation of non-Gaussian correlators (sub-scripted by "NG"),

$$
\left\langle\mathrm{e}^{\mathrm{i}^{\mathrm{Q}}\left(\eta-\eta_{0}\right.}\right\rangle_{N G},\left\langle\Delta \eta \mathrm{e}^{\mathrm{i} \mathrm{Q}_{\mathrm{z}}\left(\eta-\eta_{0}\right)}\right\rangle_{N G},\left\langle\Delta \eta \Delta \eta_{0} \mathrm{e}^{\mathrm{i} \mathrm{Q}_{\mathrm{z}}\left(\eta-\eta_{0}\right.}\right\rangle_{N G},
$$

where $\eta, \eta_{0}$ refers to the surface roughness evaluated at an arbitrary position $\boldsymbol{r}$ and at the origin, respectively. Non-Gaussian corrections to the first correlator are traditionally accounted for by a cumulant expansion,

$$
\left\langle\mathrm{e}^{\mathrm{i} \mathrm{Q}_{\mathrm{z}}\left(\eta-\eta_{0}\right)}\right\rangle_{N G} \simeq \mathrm{e}^{-Q_{z}^{2} S_{2} / 2-\mathrm{iQ}_{\mathrm{z}}^{3} \mathrm{~S}_{3} / 6+\mathrm{Q}_{\mathrm{z}}^{4} \mathrm{~S}_{4} / 24},
$$

The skewness function vanishes at the origin and remains weak over all distances. Its order of magnitude has been recently obtained in in situ conditions with help of stereo photographic technique ([32]), where it was estimated that:

$$
S_{3}(r, \phi) \sim \alpha r^{3} \cos (\phi) \exp \left(-r / r_{0}\right)
$$

with $r_{0} \sim 10^{-1}$ and $\alpha \sim 10^{-4}$. Now the leading exponential $\exp \left(-Q_{z}^{2} S_{2}\right)$ in the Kirchhoff integral is typically damped over a few EM wavelengths so that $S_{3} \sim \alpha \lambda_{E M}^{3}$ over the effective range of integration, justifying the assumption $Q_{z}^{3} S_{3} / 6 \leq 1$. Hence we can linearize the corresponding exponential and approximate:

$$
\mathrm{e}^{-Q_{z}^{2} S_{2} / 2-\mathrm{i} Q_{\mathrm{z}}^{3} \mathrm{~S}_{3} / 6+\mathrm{Q}_{\mathrm{z}}^{4} \mathrm{~S}_{4} / 24} \simeq \mathrm{e}^{-Q_{z}^{2} S_{2} / 2+Q_{z}^{4} S_{4} / 24}\left(1-\mathrm{iQ}_{\mathrm{z}}^{3} \mathrm{~S}_{3} / 6\right)
$$

Since $S_{3}$ is antisymmetric while $S_{2}$ and $S_{4}$ are centro-symmetric, the term involving $S_{3}$ vanishes after angular integration and does not contribute to the omnidirectional NRCS. We will therefore ignore the effect of the skewness function, an assumption which is further justified by the experimental and simulation results presented in [15] which shows that the upwind/downing asymmetry at moderate angles in vertical polarization is weak (less than $1 \mathrm{~dB}$ in $\mathrm{C}$ and $\mathrm{Ku}$ band). Hence the introduction of non-Gaussian characteristics in the scattering model will be limited to the excess kurtosis function $S_{4}$. We are not aware of a general non-Gaussian expansion of the second correlator in (III.20), which is implied in the $\sigma_{12}$ term of the WCA NRCS. However, the difficulty can be circumvented by performing an integration by parts,

$$
\begin{aligned}
& \frac{\mathrm{i}}{Q_{z}} \int \boldsymbol{d} \boldsymbol{r} \mathrm{e}^{-\mathrm{i} \boldsymbol{Q}_{\boldsymbol{H}} \cdot \boldsymbol{r}}\left\langle\Delta \eta \mathrm{e}^{\mathrm{i} Q_{\mathrm{Z}}\left(\eta-\eta_{0}\right)}\right\rangle= \\
& \frac{\mathrm{i}_{\mathrm{H}}^{2}}{Q_{z}^{2}} \int \boldsymbol{d} \boldsymbol{r} \mathrm{e}^{-\mathrm{i} \boldsymbol{Q}_{\boldsymbol{H}} \cdot \boldsymbol{r}}\left\langle\mathrm{e}^{\mathrm{i} \mathrm{Q}_{\mathrm{z}}\left(\eta-\eta_{0}\right)}\right\rangle-\int \boldsymbol{d} \boldsymbol{r} \mathrm{e}^{-\mathrm{i} \boldsymbol{Q}_{\boldsymbol{H}} \cdot \boldsymbol{r}}\left\langle|\nabla \eta|^{2} \mathrm{e}^{\mathrm{i} Q_{\mathrm{Z}}\left(\eta-\eta_{0}\right)}\right\rangle
\end{aligned}
$$

so that the problem amounts to calculate the non-Gaussian statistics of the simpler correlator $\left\langle|\nabla \eta|^{2} \mathrm{e}^{\mathrm{i} \mathrm{Q}_{\mathrm{z}}\left(\eta-\eta_{0}\right)}\right\rangle$. This is done in the Appendix, where it is shown that for small distances:

$\left\langle|\nabla \eta|^{2} \mathrm{e}^{\mathrm{i} \mathrm{Q}_{\mathrm{z}}\left(\eta-\eta_{0}\right)}\right\rangle \simeq\left(\operatorname{mss}-\left(1+\frac{1}{2} \gamma\right) Q_{z}^{2}|\nabla \rho|^{2}\right) \mathrm{e}^{-Q_{z}^{2} S_{2} / 2+Q_{z}^{4} S_{4} / 24}$

where $\gamma$ is the excess kurtosis of slopes. Even though this formula was established in the vicinity of the origin we will assume it is valid at arbitrary lags. This extrapolation is justified by the fact that the correlators are mainly involved by their behavior at small lags in the WCA integral. Incorporating (III.25) in (III.24) we obtain finally:

$$
\sigma_{\mathrm{omni}}^{K A}=2 \frac{|\mathbb{K}|^{2}}{Q_{z}^{2}} \int_{0}^{+\infty} J_{0}\left(Q_{H} r\right) \mathrm{e}^{-Q_{z}^{2} S_{2} / 2+Q_{z}^{4} S_{4} / 24},
$$

and

$$
\begin{aligned}
\sigma_{\mathrm{omni}}^{(12)}= & 4 \Re\left[\frac{\mathbb{K}^{*} \mathbb{T}}{Q_{z}^{2}} \int_{0}^{\infty} d r J_{0}\left(Q_{H} r\right) \mathrm{e}^{-Q_{z}^{2} S_{2} / 2+Q_{z}^{4} S_{4} / 24}\right. \\
& \left.\left(\left(1+\frac{1}{2} \gamma\right) \Delta \rho+\mathrm{mss}\right)\right]
\end{aligned}
$$

The unknown kurtosis function in the exponential is extrapolated from small to large lags as already proposed and justified in [5]:

$$
S_{4} \simeq \gamma S_{2}^{2} \exp \left(-r^{2} / r_{c}^{2}\right),
$$

where $r_{c}$ is a cut-off range defined by $K_{0}^{2} S_{2}\left(r_{c}\right)=2$. The third correlator in (III.20) seems difficult to estimate. However, it is involved in $\sigma^{(22)}$ in (III.12) which is already very small with respect to the leading two terms $\sigma^{K A}$ and $\sigma^{(12)}$. Hence, nonGaussian corrections will be simply ignored in that case.

\section{The REFERENCE DATA SETS}

The performance of the combined spectral and scattering model for the sea surface backscatter can be evaluated in the light of several available data sets. A first assessment of the models in the nadiral region has been made recently [5] with the help of a three-band $(\mathrm{C}, \mathrm{Ku}, \mathrm{Ka})$ data set and has shown the necessity to incorporate kurtosis effects. Two supplementary data sets are supplied to complete this study in $\mathrm{C}$ and $\mathrm{Ku}$ Band for larger incidence angles.

\section{A. Nadiral Data}

The following sets of near-nadir data have already been presented elsewhere [5] and will be here only briefly discussed. The first data set has been acquired by the satellite Tropical Rainfall Measuring Mission (TRMM) in $\mathrm{Ku}$ Band under various sea states and wind conditions. It covers the $0-18^{\circ}$ incidence range in horizontal polarization (which is quasi-equivalent to the vertical polarization at these low incidences). The other two data sets concerns the C band (STORM, [33]) and Ka-band (SRA, [34]) and cover a variety of wind speeds and sea states under different incidence angles (from $7^{\circ}$ to $16^{\circ}$ for STORM and $0^{\circ}$ to $15^{\circ}$ for SRA).

\section{B. Off Nadir Data}

The previous sets are complemented with two other data sets at moderate and large incidences, namely the classical NSCAT (Ku band) and the more recent ASAR ( $\mathrm{C}$ band) data sets, which are described below.

B.1. . The NSCAT instrument is a fully polarized Ku-band scatterometer (13.6 Ghz) which used to be mounted on board the ADEOS satellite. It covers a large range of incidences (18 - 54 degrees). The present data were collected during the period from May 14 to June 25, 1997 with a rich variety of wind and sea conditions. The absolute calibration of these data is not guaranteed but can be assessed by cross-comparison with 24 the more recent TRMM instrument at the common available incidence (18 degrees), as shown on Figure 2. A difference of about $0.8 \mathrm{~dB}$ is observed between the instruments. This gives the expected level of uncertainty in the magnitude of the NRCS used in our simulations. 

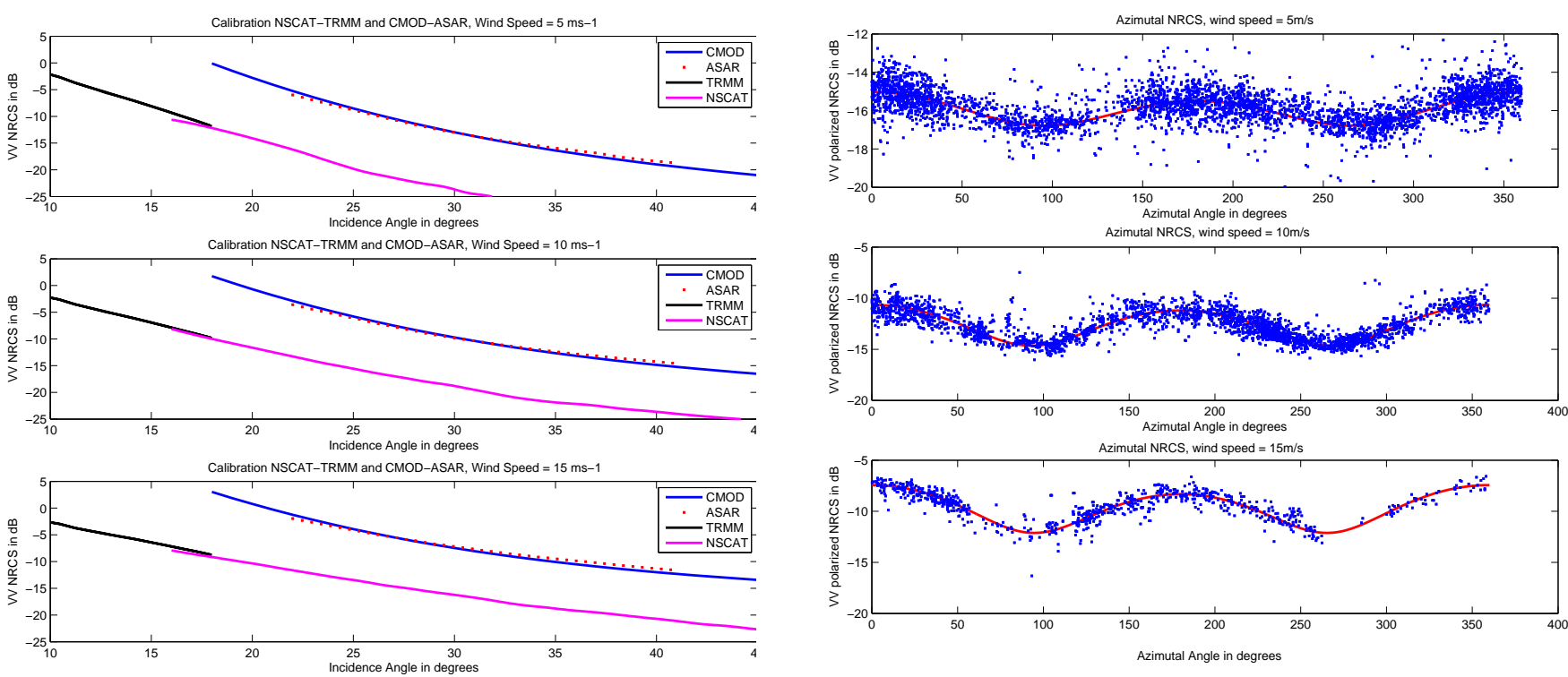

Fig. 2. Consistency of different sets in $\mathrm{C}(\mathrm{CMOD} / \mathrm{ASAR})$ and $\mathrm{Ku}$ (TRMM/NSCAT) data at different wind speeds.

B.2. . ASAR stands for "Advanced Synthetic Aperture Radar" and is one the instruments carried by the ENVISAT satellite. This $\mathrm{C}$ Band $(6 \mathrm{GHz})$ radar is capable of working in alternating polarization, thus providing the simultaneous vertical and horizontal backscatter with the same instrument. The vertical polarization has been cross-calibrated with comparison with the classical CMOD-5 model [35], as shown on Figure 2. The current data set has been acquired during a period of four weeks in 2005 and four weeks in 2007. A direct treatment of the level 0 raw product makes it possible to evaluate and subtract the contribution of additive instrumental noise. The NRCS is measured for incidence angles ranging from $20^{\circ}$ to $45^{\circ}$ under various wind conditions and sea states. Instantaneous wind speed and direction of the satellite track is estimated by ASAR and also by the collocated scatterometer ASCAT [36]. For this study, we will use the wind speed estimation by the scatterometer which it is assumed to be more accurate. The NRCS in different polarizations have been sorted by bins of wind speed and direction and incidence angles. However, a large dispersion on the level of NRCS is still observed. This variability has been smoothed out by a least square regression which best follows the azimuthal variations of the NRCS. The latter are have been sought as usual in Fourier series of the azimuth angle with two harmonics:

$$
\sigma^{0}=a_{0}+a_{1} \cos \phi+a_{2} \cos 2 \phi
$$

where $\sigma^{0}$ is the NRCS, $a_{0}, a_{1}$ and $a_{2}$ are the coefficients of the regression and $\phi$ is the wind direction. This expansion has been done at each incidence angle and wind speed. Hence, $a_{0}, a_{1}$ and $a_{2}$ are functions of the incidence angles and wind speed. As an example, Figure 3 shows the azimuthal variations of the raw (blue points) and smoothed (red solid line) NRCS at $35^{\circ}$ and $5 \mathrm{~ms}^{-1}$ wind speed. A further smoothing over incidence angles is performed on the coefficients $a_{0}, a_{1}$ and $a_{2}$ with an angled window of 2 degrees.

It is important to note that the absolute level of the radar cross-section is necessary for the estimation of the curvature spectrum. This was not the case for nadiral data for which only
Fig. 3. Variation of the ASAR vertically polarized NRCS with azimuth $\phi$ at 35 degrees incidence by three wind speeds : 5,10 and $15 \mathrm{~ms}^{-1}$.

the relative NRCS (with respect to their maximum) was needed to estimate global parameters such as the mss or the kurtosis of slopes. Hence the nadiral data are free of calibration issues while the off-nadir might suffer from some bias. However, the data set we use (ASAT, NSCAT) have been cross-calibrated with other instruments and will be assumed reliable, even through a value for their absolute accuracy cannot be given.

\section{Performances of existing spectra}

\section{A. Nadiral region}

The performances of the reference existing spectra in terms of consistency with the experimental data sets can be evaluated with the help of the WCA scattering model. In the nadiral region the NRCS is quasi unpolarized and is accurately described by the mere Kirchhoff approximation $\sigma^{K A}$ (the corrective terms brought by WCA are negligible here). Simulations of the omnidirectional NRCS performed with the reference classical spectra (Elfouhaily and Kudryavtsev) and the KA under a linear sea surface assumption (i.e. the first term in (III.16) show significant differences with the experimental NRCS. As an example, Figure 4 shows the omnidirectional NRCS in different frequency bands at $5 \mathrm{~ms}^{-1}$ wind speed according to Elfouhaily spectrum. A comparison is done with available data (SOWEX, TRMM, STORM). To eliminate possible calibration errors, only the relative NRCS is shown (that is the NRCS divided by its maximum value) with an offset of $3 \mathrm{~dB}$ (Ku band) and $12 \mathrm{~dB}$ (Ka band) to clarify the plot. As seen, Elfouhaily spectrum provides a reasonable agreement with the nadiral data except in Ka band, while Kudryavtsev spectrum (not shown here) overestimates the relative NRCS by a few dBs. As was shown in [5], the introduction of a slope kurtosis parameter improves the simulation of the nadiral NRCS. The non-Gaussian KA according to (III.26) and (III.28) is shown on the same Figure 4 with an optimized value of the excess kurtosis parameter $(\gamma=0.19$ for $5 \mathrm{~m} / \mathrm{s}$ wind speed). This brings the simulated NRCS closer to the data but is not sufficient to ensure a good matching in Ka band. 


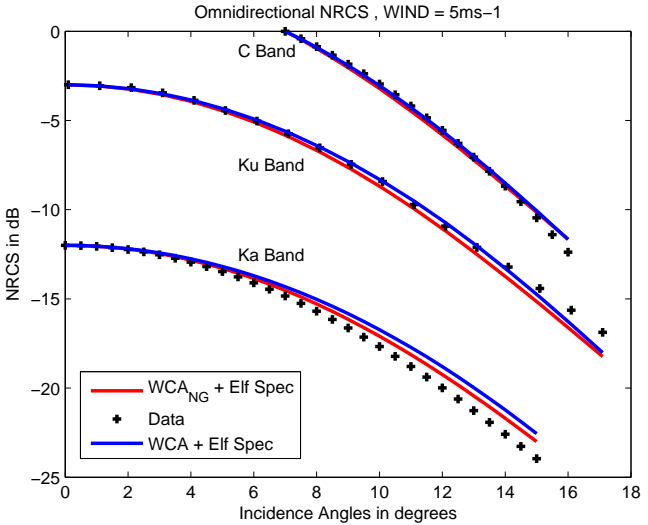

Fig. 4. Simulated omnidirectional VV NRCS using Elfouhaily spectrum in the Kirchhoff model under a Gaussian (KA/WCA) and nonGaussian (KA/WCA-NG) assumption by a wind speed of $5 \mathrm{~ms}^{-1}$. The NRCS is taken relative to its maximum value. A further offset of $3 \mathrm{~dB}$ ( $\mathrm{Ku}$ band) and $12 \mathrm{~dB}$ ( $\mathrm{Ka}$ band) is applied for better visibility.

\section{B. Off-nadir incidences}

Figure 5 shows the simulated (absolute) NRCS in the Gaussian and non-Gaussian WCA at moderate and large incidences and a comparison with the available off-nadir data sets. An off-set of $15 \mathrm{~dB}$ has been applied to the $\mathrm{Ku}$ band for the clarity of the plot. Again the Elfouhaily omnidirectional spectrum has been used. Even though it gives far better results than Kudryavtsev model (not shown here), it is still 2-3 dB away from $\mathrm{C}$ band data and the non-Gaussian correction is not sufficient to correct for the discrepancy.

To evaluate the impact of the scattering model, other methods have been tested and superimposed to the WCA results, namely the three-scale model [27] (whose code was kindly provided to us by Dr Plant) and the second-order Small-Slope Approximation (SSA2, [37]). For this last method, we reported values from [38] for the upwind and crosswind NRCS after Elfouhaily directional spectrum and assumed that the omnidirectional NRCS is the half-sum of the latter (this is actually true if the NRCS is composed of two azimuthal harmonics, $\left.\sigma^{0}=a_{0}+a_{2} \cos (2 \phi)\right)$. The NRCS values derived from the different scattering models agree within $1 \mathrm{~dB}$, which is far less than the observed discrepancy with data.

The same qualitative conclusions hold for different wind speeds and spectra, even through the scattering models may perform well at higher wind speeds some (but not all) bands, as for instance Elfouhaily spectrum in $\mathrm{Ku}$ band. A non-Gaussian scattering model incorporating both a realistic excess kurtosis of slope and an improved shape of the omnidirectional spectrum are thus necessary to simulate the omnidirectional NRCS in a consistent way with respect to the experimental data. In the following, the curvature spectrum as well as the kurtosis parameter will be sought and optimized using the non-Gaussian WCA model.

\section{Parametrization of the SPECtrum}

As shown in the previous section, none of the mentioned classical spectra can allow for a consistent estimation of the omnidirectional NRCS whenever several bands and incidences are considered simultaneously, even with an improved scattering model. In addition they do not fully meet the a priori conditions imposed by the total and filtered mss inferred from optical measurements. It is therefore necessary to devise a modified om-

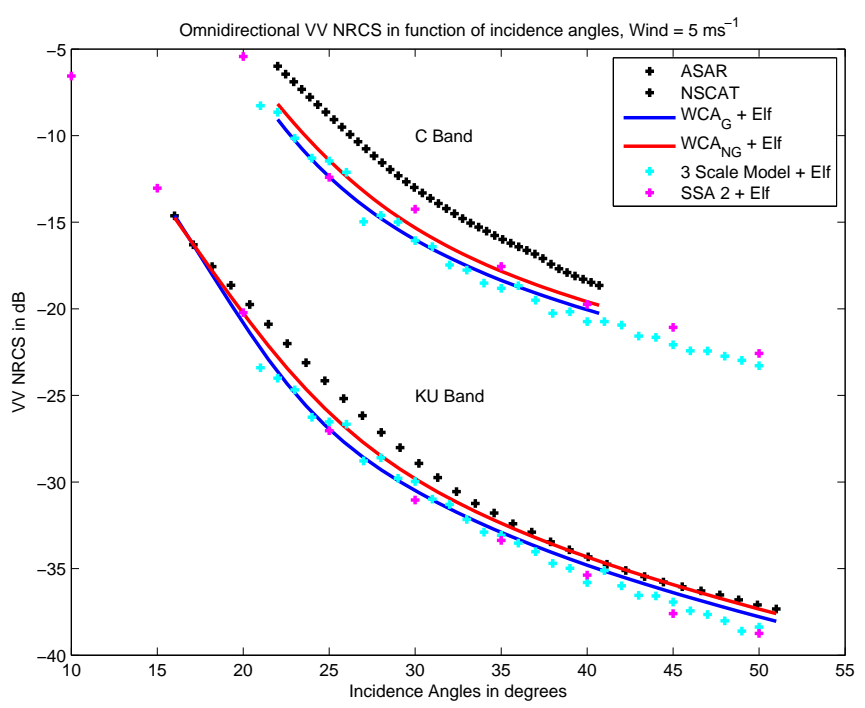

Fig. 5. Simulated omnidirectional absolute VV NRCS using Elfouhaily omnidirectional spectrum in the WCA model under a Gaussian (WCA) and non-Gaussian (WCA-NG) assumption by a wind speed of $5 \mathrm{~ms}^{-1}$. A comparison is also given with SSA2 and Three-scale model using the same spectrum. An off-set of $15 \mathrm{~dB}$ has been applied to the $\mathrm{Ku}$ band for better visibility.

nidirectional spectrum in the short-wave domain to correct for these shortcomings. As it is customary we will use the omnidirectional curvature spectrum,

$$
B(k)=k^{3} S(k)
$$

rather than the omnidirectional spectrum itself in order to compensate the overall $k^{-3}$ decay which masks the actual spectral shape. As commonly employed we will split this quantity into a long wave $\left(B_{l w}\right)$ and short-wave part $\left(B_{s w}\right)$ :

$$
B(k)=B_{l w}(k)+B_{s w}(k)
$$

The scattering data in the microwave regime are mostly sensitive to the shape of the short-wave spectrum which we will seek to optimize. For the long-wave spectrum we will take a generic wind-dependent parametric model in open sea. We will ignore the coupling between long and short-wave spectrum and the modification induced by swell [39], [40] or dominant waves [41] on the latter. This is probably a limitation for specific sea conditions but remains relevant in the search of a "mean" spectrum (with respect to all possible sea states under a given wind) which cannot include these additional effects. In the numerical simulations the low-frequency part of the omnidirectional sea spectrum will thus be simply taken identical to the unified model of Elfouhaily with an overall multiplicative factor $\alpha$ to make it consistent with the filtered slopes measured in [24].

$$
B_{l w}(k)=\alpha B_{E}(k), k \leq k_{1},
$$

where $B_{E}$ is the omnidirectional curvature spectrum described in [3]. The factor $\alpha$ is defined by:

$$
\alpha=\frac{\operatorname{mss}_{l}}{\int_{0}^{k_{1}} B_{E}(k) k^{-1} d k},
$$

where $\operatorname{mss}_{l}$ is the the slope of one-meter segments on the surface, as measured in [24] by means of three one-meter spaced 
vertical lasers. This quantity is interpreted as the mss of scales larger than 1-2 meters, corresponding to a limiting wave number $k_{1}$. A more precise estimation of this value can be obtained by integrating a reference long-wave spectrum and tuning the cut-off parameter $k_{1}$ to make it coincide with the given large scale mss:

$$
\operatorname{mss}_{l}=\int_{0}^{k_{1}} B_{E}(k) k^{-1} d k .
$$

This provides an estimate $k_{1} \simeq 2 \pi / 1.6 \mathrm{rad} / \mathrm{m}$ depending on wind speed.

The short-wave curvature $B_{s w}$ is sought as a simple parametric function of the wave number $k$ for $k \geq k_{1}$. The most salient feature of the Elfouhaily curvature spectrum is a sharp peak at a certain wavenumber $\left(k_{3}=363 \mathrm{rad} / \mathrm{m}\right)$ corresponding to the minimum phase speed $\left(c_{m}\right)$ :

$$
B_{\text {sw, Elfouhaily }}=\frac{1}{2} \frac{c_{m}}{c} \exp \left(-\frac{1}{4}\left(\frac{k}{k_{3}}-1\right)\right)
$$

This peak is essential to capture the physical mechanism of energy accumulation occurring around this wavenumber. The existence of this peak will be conserved in the parametric model but its position $\left(k_{3}\right)$, shape and magnitude will be relaxed and subject to a certain degree of freedom. In addition it will be possibly asymmetric and wind dependent. To this aim the omnidirectional curvature will be sought in the form:

$$
B_{s w}(k)=\left\{\begin{array}{l}
C \mathrm{e}^{-g_{2}\left(\log _{10}\left(\frac{k}{k_{3}}\right)\right)^{2}}, k_{2} \leq k \leq k_{3}, \\
C \mathrm{e}^{-g_{3}\left(\left(\frac{k}{k_{3}}\right)^{2}-1\right)}, k \geq k_{3}
\end{array}\right.
$$

where $C, k_{3}, g_{2}, g_{3}$ are free parameters and $k_{2}$ is the crossing point of the two functions. The Gaussian shape of the curvature spectrum for $k \geq k_{3}$ is kept identical to that proposed in Elfouhaily model and ensures a sharp cut-off due to viscous damping above a critical wavenumber. The left side of the peak, however, is modified to produce a smoother decay at lower wavenumbers. The intermediate wavenumbers are described by the right half of a Gaussian starting at $k_{1}$ :

$$
B_{s w}(k)=B_{l w}\left(k_{1}\right) \mathrm{e}^{-g_{1}\left(\log _{10}\left(\frac{k}{k_{1}}\right)\right)^{2}}, k_{1} \leq k \leq k_{2} .
$$

The reason for the occurrence of a decreasing function in this range of wavenumbers is the following observation. The slick and clean mss predicted by Elfouhaily spectrum are larger than those experimentally observed by Phillips [23] and CM. On the other hand the large scale mss calculated for Elfouhaily spectrum is consistent with the measurements by [24]. Since Elfouhaily omnidirectional curvature spectrum is almost constant in the range of wavenumbers corresponding to decimeter scales, this means that the actual curvature spectrum has an excavation with respect to the latter in this same range of wavenumber to ensure a correct estimate of the slick mss.

\section{INVERSION OF THE SPECTRUM}

The spectral parameters $C, g_{1}, g_{2}, g_{3}, k_{3}$ as well as the kurtosis parameter $\gamma$ have been numerically optimized in order for the simulated NRCS to match as best the full set of experimental data. This consists in minimizing the following cost function:

$$
\langle|\left(\sigma_{\text {omni }}^{0}(\text { model })\right)_{d B}-\left(\sigma_{\text {omni }}^{0}(\text { data })\right)_{d B}|\rangle
$$

where the average is performed over all available incidences and bands and the NRCS are taken in $\mathrm{dB}$ prior to the substraction. As before relative NRCS are taken at near-nadir angles

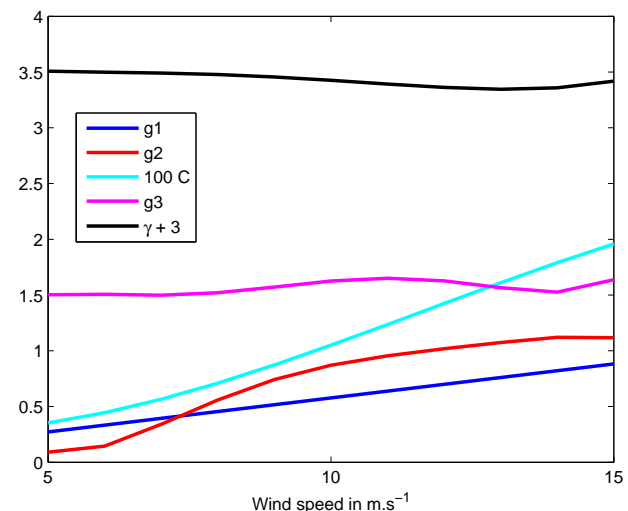

Fig. 6. Evolution of the different parameters of the inverted spectrum as a function of wind speed

and absolute values at off-nadir angles and the same weight is applied to each available data set, so that the cost function is robust to the incidence binning. The calculation of the coefficients has been performed with the help of the Matlab routine lsqnonlin ([42]). This function solves a non-linear minimization problem based on the least square method for the set of entry parameters. The evolution of the different parameters with wind speed is shown on Figure 6. In view of their smooth variations, these functions can be accurately described by low-order polynomial approximations of the wind speed variable $u=U_{10}$. The following expressions are found in the range $u=[5-15]$ $m s^{-1}$ (the minimum number of significant digits is given for the coefficients).

$$
\begin{aligned}
g_{1} & =6.110^{-2} u-3.310^{-2} \\
g_{2} & =-1.31410^{-4} u^{5}+7.03610^{-3} u^{4}-1.46810^{-1} u^{3} \\
& +1.4741 u^{2}-6.9122 . u+12.162 \\
g_{3} & =1.537810^{-4} u^{5}-7.180410^{-3} u^{4}+1.283510^{-1} u^{3} \\
& -1.095 u^{2}+4.4721 u-5.52 \\
C= & -8.610^{-6} u^{3}+310^{-4} u^{2}-1.610^{-3} u+5.110^{-3} \\
\gamma & =1.48610^{-4} u^{4}-510^{-3} u^{3}+5.8610^{-2} u^{2} \\
& -2.9910^{-1} u+1.07
\end{aligned}
$$

The high-wavenumber cut-off parameter is found independent of wind speed and equal to $k_{3}=308$. The excess kurtosis of slopes reaches a minimum value 0.36 at $12 \mathrm{~ms}^{-1}$. This qualitative behavior has already been observed experimentally in [24]. The mixing effect of wind renders the surface statistics more Gaussian-like until it reaches a state where breaking phenomena and sharp crests become important and deviate from the Gaussian statistics.

The omnidirectional curvature spectrum is plotted on Figure 7 together with the reference Elfouhaily model. The main qualitative novelties are an excavation of the curvature spectrum at intermediate scales and a slight downshift of the peak position. The total and partial mss of the inverted spectrum are shown on Figure 8 as a function of wind speed. By construction the calculated large scale mss is consistent with the experimental observations. The total and slick mss are in very good agreement with CM values (within $5 \%$ error) except at small wind speed where they are slightly overestimated (by about $15 \%$ ). Note that these 


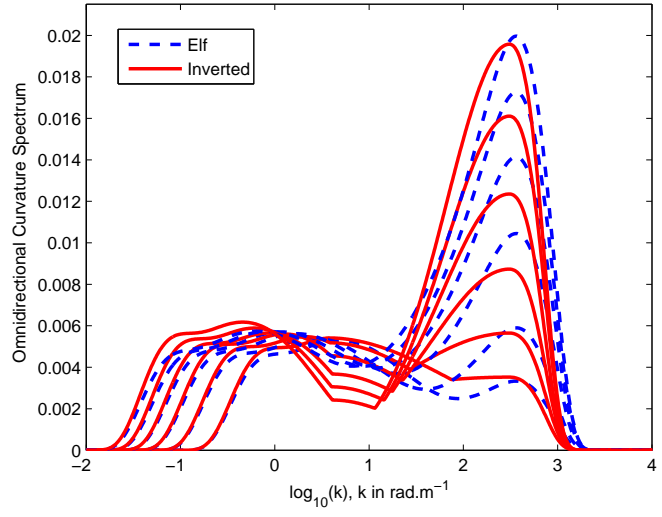

Fig. 7. Evolution of the inverted omnidirectional curvature spectrum (inverted) with wind speed. Elfouhaily model (Elf) is given for reference.

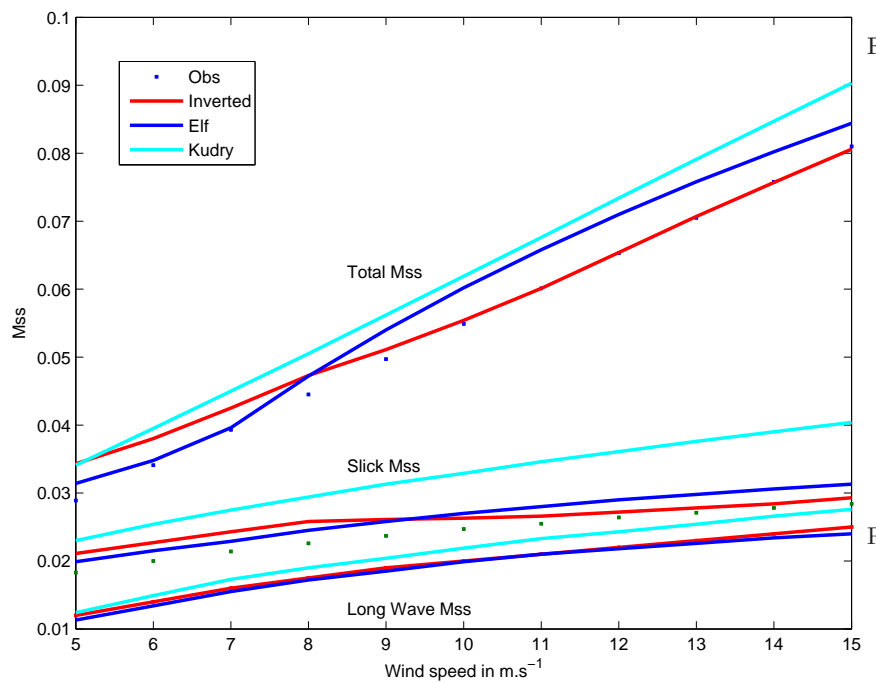

Fig. 8. Total and filtered mss calculated from the inverted spectrum as a function of wind speed. A comparison is provided with Elfouhaily (Elf) and Kudryavtsev (Kudry) models as well as experimental observations (Obs).

values result from the integration of the new omnidirectional curvature spectrum, obtained itself from an optimization of the sole NRCS with no a priori constraints on the total and slick mss. The good agreement observed with the latter experimental values at moderate wind speeds confirm the correctness of the novel omnidirectional curvature spectrum. The slight discrepancy observed at small wind speeds is explained by the lesser quality of the matching of simulated and experimental NRCS data, especially in $\mathrm{C}$ band. A possible explanation for this could be the inability of the current surface scattering model to capture additional non-Gaussian phenomena such as those caused by the variability of the statistics from one patch to another [10]. Figure 9 shows the evolution of the partial mss as function of the high-frequency wavenumber at different wind speeds. The median wavenumbers, that is the values at which half of the total mss is attained, are marked by red crosses. Contrarily to the common expectation the median wavenumber is shifted to the right as wind increases. This shows that short scales have an increasing contribution to the total mss even through there

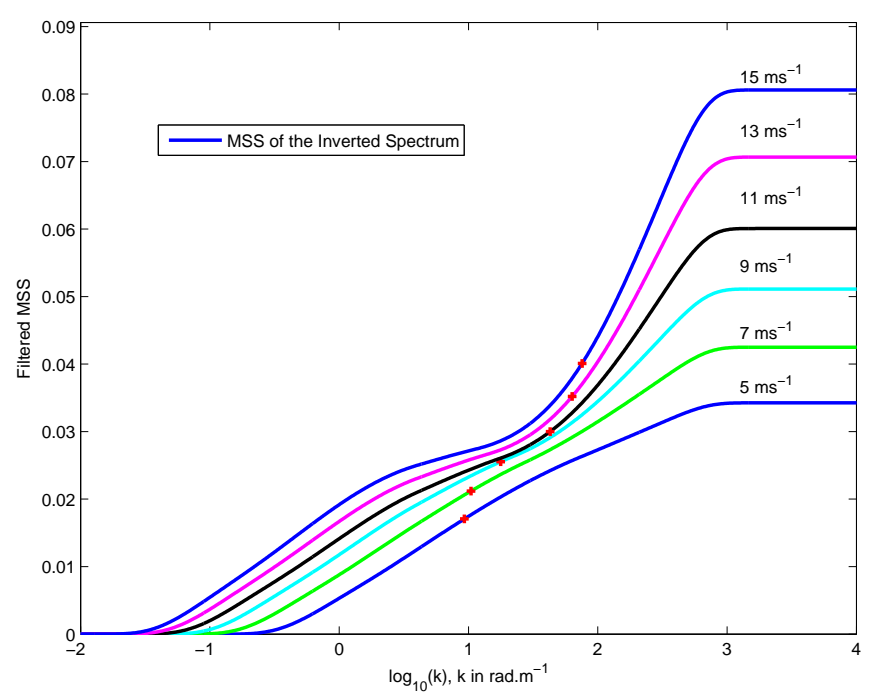

Fig. 9. Evolution of the filtered mss as a function of the high-wavenumber cut-off. Half of the total mss is reached at the crosses.

\begin{tabular}{|c||c||c||c||c||c||c|}
\hline & $\mathbf{C}$ & & $\mathbf{K u}$ & & Ka & \\
ws & NOS & Elf & NOS & Elf & NOS & Elf \\
\hline 5 & $\mathbf{1 . 2 4}$ & 2.68 & $\mathbf{0 . 5 2}$ & 1.30 & $\mathbf{0 . 3 5}$ & 0.40 \\
\hline 7 & $\mathbf{0 . 7 8}$ & 2.09 & $\mathbf{0 . 5 4}$ & 0.99 & 0.49 & $\mathbf{0 . 4 3}$ \\
\hline 9 & $\mathbf{0 . 6 1}$ & 0.90 & $\mathbf{0 . 4 0}$ & 0.66 & 0.25 & $\mathbf{0 . 1 9}$ \\
\hline 11 & $\mathbf{0 . 6 4}$ & 0.76 & $\mathbf{0 . 4 2}$ & 0.52 & 0.21 & $\mathbf{0 . 1 0}$ \\
\hline 13 & $\mathbf{0 . 8 7}$ & 1.24 & 0.34 & $\mathbf{0 . 2 2}$ & $\mathbf{0 . 4 0}$ & 0.42 \\
\hline 15 & $\mathbf{0 . 9 0}$ & 1.38 & 0.46 & $\mathbf{0 . 3 7}$ & $\mathbf{0 . 2 4}$ & 0.30 \\
\hline
\end{tabular}

TABLE I

Performances of the SCATtering Model USing the NeW OMNIDirectional SPECTRUM (NOS) IN THE DIFFERENT BANDS. A COMPARISON IS GIVEN WITH

Elfouhaily MOdel (ELF)

is a constant increase of large scale mss with growing dominant wave.

\section{QuALity OF THE INVERSION}

The quality of the inversion can be assessed using the same criterion as previously, with the refinement that the cost function (VII.38 ) can be calculated separately over different bands. Results are summarized in Table III. As can be shown, the new spectrum allows for an overall better reconstruction of the omnidirectional NRCS, with a marked improvement in $\mathrm{C}$ band and $\mathrm{Ku}$ band by small winds and equivalent performances otherwise. The performances of the model in the different bands is examplified on Figure 10 at $5 \mathrm{~ms}^{-1}$ wind speed. The vertically polarized NRCS is shown as a function of incidence and compared with available data sets (an off-set of $10 \mathrm{~dB}$ has been applied to $\mathrm{Ku}$ data for clarity). As can be seen, a significant improvement over Elfouhaily model is obtained for off-nadir incidences in $\mathrm{C}$ and $\mathrm{Ku}$ bands.

\section{Conclusion}

This work addressed the issue of consistent simulation of microwave back-scattering from the sea surface whenever several bands and incidences are considered. It has set a methodology for the construction of a pure "remote sensing spectra". When 


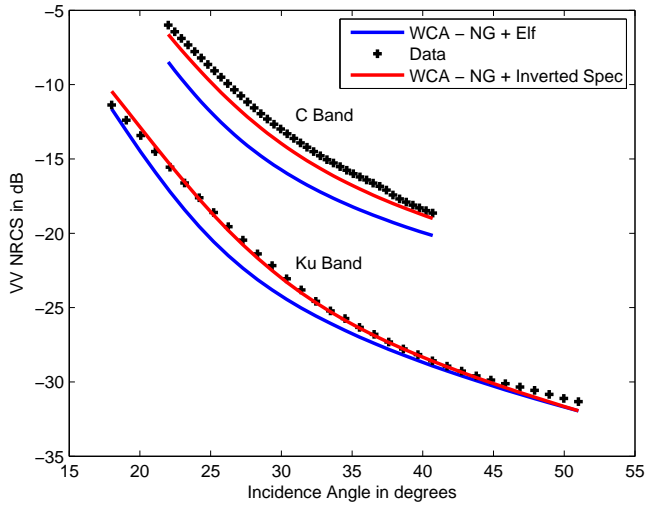

Fig. 10. Simulation of the omnidirectional VV NRCS according to the inverted omnidirectional spectrum and the non-Gaussian WCA model (WCA-NG+inverted spec) by a $5 \mathrm{~ms}^{-1}$ wind speed. A comparison is given with available data and simulations according to the Elfouhaily model (WCA-NG+Elf). An off-set of $10 \mathrm{~dB}$ has been applied to $\mathrm{Ku}$ data for clarity.

off-nadir angles are considered, the level of the short-wave spectral curvature is a very discriminating parameter for the NRCS which renders possible an inversion procedure from the latter. The main outcome of this study is that at least two conditions are necessary to bring simulations closer to the experimental observations. First, a non-Gaussian scattering model should be employed, as was already emphasized elsewhere for the nadiral region [5]. Second, inversion of the curvature from the multiband data sets reveals important differences with the classical spectra, in particular at intermediate scales $(20 \mathrm{~cm}-2$ meter $)$ where a pronounced dip of energy is observed. Further investigations are necessary to confirm and refine this marked result with the help of most complete data sets. The restrictive assumptions which have been adopted for this first study (omnidirectional NRCS, vertically polarized data) should be relaxed. In particular, directional aspects, fully polarized data and absolute calibration issues should be considered. Also, our present findings should be consolidated by other bands and data sets, in particular $\mathrm{L}$ band to refine the decimeter range of the spectrum where a dip is observed, especially by small winds where the calculated mss has been found less accurate. These questions, however, go beyond the scope of this paper and are left for future research.

\section{APPENDIX}

The correlator $\left\langle|\nabla \eta|^{2} \mathrm{e}^{\mathrm{i} \mathrm{Q}_{\mathbf{z}}\left(\eta-\eta_{0}\right)}\right\rangle$ is explicit in the Gaussian case,

$$
\left\langle|\nabla \eta|^{2} \mathrm{e}^{\mathrm{i} \mathrm{Q}_{z}\left(\eta-\eta_{0}\right)}\right\rangle=\left(\operatorname{mss}-Q_{z}^{2}(\nabla \rho)^{2}\right) \mathrm{e}^{-Q_{z}^{2} S_{2} / 2}
$$

but is otherwise unknown. However, a relevant approximation can be established in the non-Gaussian case using a cumulant expansion. To simplify the approach we will first consider the one-dimensional case $\left(\eta(\boldsymbol{r}) \rightarrow \eta(x), \nabla \eta(\boldsymbol{r}) \rightarrow \eta^{\prime}(x), \nabla \rho \rightarrow\right.$ $\rho^{\prime}, \operatorname{mss}^{\prime} \operatorname{mss}_{x}$ ) and will discard the skewness terms (that is third-order moments involving $\eta-\eta_{0}$ and $\eta^{\prime}$ ). We first observe that:

$$
\left\langle\eta^{\prime 2} \mathrm{e}^{\mathrm{i} \mathrm{Q}_{\mathrm{Z}}\left(\eta-\eta_{0}\right)}\right\rangle=-\left[\frac{\partial^{2}}{\partial \alpha^{2}}\left\langle\mathrm{e}^{\mathrm{i} \mathrm{Q}_{\mathrm{z}}\left(\eta-\eta_{0}\right)+\mathrm{i} \alpha \eta^{\prime}}\right\rangle\right]_{\alpha=0}
$$

Now performing a cumulant expansion of the random variable $X=Q_{z}\left(\eta-\eta_{0}\right)+\alpha \eta^{\prime}$,

$$
\log \left\langle\mathrm{e}^{\mathrm{iX}}\right\rangle=-\frac{1}{2}\left\langle X^{2}\right\rangle+\frac{1}{24}\left(\left\langle X^{4}\right\rangle-3\left\langle X^{2}\right\rangle^{2}\right)+\ldots
$$

we obtain:

$$
\left\langle\eta^{\prime 2} \mathrm{e}^{\mathrm{i} \mathrm{Q}_{\mathrm{z}}\left(\eta-\eta_{0}\right)}\right\rangle=-\left(A_{1}^{2}+2 A_{2}\right) \mathrm{e}^{A_{0}},
$$

with

$$
\begin{aligned}
& A_{0}=-\frac{1}{2} Q_{z}^{2} S_{2}+\frac{1}{24} S_{4} \\
& A_{1}=Q_{z} \rho^{\prime}+\frac{1}{6} Q_{z}^{3}\left(\left\langle\left(\eta-\eta_{0}\right)^{3} \eta^{\prime}\right\rangle-3 \rho^{\prime} S_{2}\right) \\
& A_{2}=-\frac{1}{2} \operatorname{mss}_{x}+\frac{1}{4} Q_{z}^{2}\left(\left\langle\left(\eta-\eta_{0}\right)^{2}\left(\eta^{\prime}\right)^{2}\right\rangle-2 \rho^{\prime 2}-\operatorname{mss}_{x} S_{2}\right)
\end{aligned}
$$

Approximating the involved functions by their leading term at the origin we obtain more simply:

$$
\begin{aligned}
& A_{0}=-\frac{1}{2} Q_{z}^{2} S_{2}+\frac{1}{24}\left(S_{4}-3 S_{2}^{2}\right) \\
& A_{1}=Q_{z} \rho^{\prime} \\
& A_{2}=-\frac{1}{2} \operatorname{mss}_{x}+\frac{\gamma}{4} Q_{z}^{2} \operatorname{mss}_{x} x^{2} \simeq-\frac{1}{2} \operatorname{mss}_{x}+\frac{\gamma}{4} Q_{z}^{2} \rho^{\prime 2}
\end{aligned}
$$

where $\gamma$ is the excess kurtosis of slopes. The extension to a $2 \mathrm{D}$ isotropic process is straightforward, leading to:

$\left\langle|\nabla \eta|^{2} \mathrm{e}^{\mathrm{i} \mathrm{Q}_{\mathrm{z}}\left(\eta-\eta_{0}\right)}\right\rangle \simeq\left(\operatorname{mss}-\left(1+\frac{1}{2} \gamma\right) Q_{z}^{2}|\nabla \rho|^{2}\right) \mathrm{e}^{-Q_{z}^{2} S_{2} / 2+Q_{z}^{4} S_{4} / 24}$,

As expected the formula reduces to (.40) in the Gaussian case $\left(\gamma=0, S_{4}=0\right)$.

\section{REFERENCES}

[1] OM Phillips. The equilibrium range in the spectrum of wind-generated waves. J. Fluid Mech, 4(4):426-434, 1958.

[2] W.J. Pierson Jr and L. Moskowitz. A proposed spectral form for fully developed wind seas based on the similarity theory of sa kitaigorodskii. Journal of geophysical research, 1964.

[3] T. Elfouhaily, B. Chapron, K. Katsaros, and D. Vandemark. A unified directional spectrum for long and short wind-driven waves. J. Geophys. Res., 102(C7):15781-15796, July 1997.

[4] V.N. Kudryavtsev, V.K. Makin, and B. Chapron. Coupled sea surfaceatmosphere model,2, Spectrum of short wind waves. Journal of geophysical research, 104, 1999

[5] A. Bringer, C-A. Guérin, B. Chapron, and A. Mouche. Peakedness effects in near-nadir radar observations of the sea surface. IEEE Trans. Geosci. and Remote Sens., 2012. to appear.

[6] R. Lawner and R. Moore. Short gravity and capillary wave spectra from tower-based radar. Oceanic Engineering, IEEE Journal of, 9(5):317-324, 1984.

[7] T.M. Elfouhaily and C.A. Guérin. A critical survey of approximate scattering wave theories from random rough surfaces. Waves in Random and Complex Media, 14(4):1-40, 2004.

[8] K.S. Chen, A.K. Fung, and D.E. Weissman. A backscattering model for ocean surfaces, IEEE Trans, Geosci, and Remote Sens, 30(4):811-817, 1992.

[9] K.S. Chen, A.K. Fung, and F. Amar. An empirical bispectrum model for sea surface scattering. IEEE Trans. Geosci. and Remote Sens., 31(4):830835, 1993.

10] B. Chapron, V. Kerbaol, D. Vandemark, and T. Elfouhaily. Importance of peakedness in sea surface slope measurements and applications. Journal of Geophysical Research-Oceans, 105(C7), 2000

[11] Y. Liu, M.Y. Su, X.H. Yan, and W.T. Liu. The mean-square slope of ocean surface waves and its effects on radar backscatter. J. Atmos. Oceanic Tech., $17(8): 1092-1105,2000$

[12] C.-A. Guérin. Scattering on rough surfaces with alpha-stable non-Gaussian height distributions. Waves in Random and Complex Media, 12(3):293-306, 2002.

[13] G. Engen, I. Friestad-Pedersen, H. Johnsen, and T. Elfouhaily. Curvature effects in ocean surface scattering. IEEE Trans. Antennas Propag., 54(5):1370-1379, 2006 
[14] C. Bourlier. Azimuthal harmonic coefficients of the microwave backscattering from a non-gaussian ocean surface with the first-order ssa model. IEEE Trans. Geosci. and Remote Sens., 42(11):2600-2611, 2004.

[15] AA Mouche, B. Chapron, N. Reul, D. Hauser, and Y. Quilfen. Importance of the sea surface curvature to interpret the normalized radar cross section. Journal of Geophysical Research, 112(C10), 2007.

[16] F. Nouguier, C.-A. Guérin, and B. Chapron. Scattering From Nonlinear Gravity Waves: The "Choppy Wave" Model. IEEE Trans. Geosci. and Remote Sens., 48(12):4184-4192, 2010.

[17] F. Nouguier, C.A. Guérin, and G. Soriano. Analytical techniques for the doppler signature of sea surfaces in the microwave regime- ii: Nonlinear surfaces. Geoscience and Remote Sensing, IEEE Transactions on, 49(12):4920-4927, 2011.

[18] C.A. Guérin, G. Soriano, and B. Chapron. The Weighted Curvature Approximation in scattering from sea surfaces. Waves in Random and Complex Media, 20(3):364-384, 2010 .

[19] J. W. Wright. A new model for sea clutter. IEEE Trans. Antennas Propagat., 16:217-223, March 1968 .

[20] G. Soriano and C-A. Guérin. A cutoff invariant two-scale model in electromagnetic scattering from sea surfaces. IEEE Geoscience and Remote Sensing Letters, 5(2):199, 2008.

[21] B. Jähne and K.S. Riemer. Two-dimensional wave number spectra of smallscale water surface waves. J. Geophys. Res., 95(C7):11.531-11.546, 1990.

[22] C. Cox and W. Munk. Statistics from the sea surface derived from the sun glitter. J. Marine Res., 13:198-227, 1954.

[23] O.M. Phillips. The dynamics of the upper ocean. Cambridge university press, 1977

[24] D. Vandemark, B. Chapron, J. Sun, G.H. Crescenti, and H.C. Graber. Ocean wave slope observations using radar backscatter and laser altimeters. J. Phys. Oceanogr., 34:2825-2842, december 2004.

[25] Sébastien Guimbard. Interprétation et modélisation de mesures à distance de la surface marine dans le domaine micro-onde. $\mathrm{PhD}$ thesis, Université Versailles-Saint-Quentin-en-Yvelines, 2010.

[26] A. Voronovich. Small-slope approximation for electromagnetic wave scattering at a rough interface of two dielectric half-spaces. Waves in Random and Complex Media, 4(3):337-367, 1994

[27] W.J. Plant. A stochastic, multiscale model of microwave backscatter from the ocean. Journal of geophysical research, 107(C9):3120, 2002.

[28] T. Elfouhaily, S. Guignard, R. Awadallah, and D.R. Thompson. Local and non-local curvature approximation: a new asymptotic theory for wave scattering. Waves in Random and Complex Media, 13(4):321-337, 2003.

[29] A. Mouche, B. Chapron, and N. Reul. A simplified asymptotic theory for ocean surface electromagnetic wave scattering. Waves in Random and Complex Media, 17(3):321-341, 2007.

[30] C.A. Guérin, G. Soriano, and T. Elfouhaily. Weighted curvature approximation: numerical tests for $2 \mathrm{~d}$ dielectric surfaces. Waves in random media, 14(3):349-363, 2004.

[31] AK Fung and KS Chen. Kirchhoff model for a skewed random surface. Journal of Electromagnetic Waves and Applications, 5(2):205-216, 1991.

[32] A. Mironov, D. Hauser, M. Kosnik, V. Dulov, and CA Guerin. Statistical characterization of short wind-waves from stereo images of the sea surface. Journal of Geophysical Research, 117:C00J35, 2012.

[33] D. Hauser, A. Mouche, B. Chapron, H. Johnsen, J.F. Daloze, C. Guerin, G. Caudal, and J.M. Lefevre. Ocean wave spectrum and radar cross-section analysis from coincident envisat asar observations and airborne polarimetric radar measurements performed during the valpareso experiment. In Geoscience and Remote Sensing Symposium, 2003. IGARSS'O3. Proceedings. 2003 IEEE International, volume 3, pages 1910-1914. IEEE, 2003.

[34] E.J. Walsh, M.J. Banner, C.M Wrigth, D.C Vandermark, B Chapron, J Jensen, and L. See. The Southern Ocean Waves Experiment. Part III: Sea Surface Slope Statistics and Near-Nadir Remote Sensing. Journal of Physical Oceanography, 38:670-684, 2008.

[35] Y. Quilfen, B. Chapron, T. Elfouhaily, K. Katsaros, and J. Tournadre. Observation of tropical cyclones by high-resolution scatterometry. Journal of Geophysical Research, 103(C4):7767-7786, 1998.

[36] Ocean and Sea Ice SAF. ASCAT Wind Product User Manual, 2011. Version 1.10 .

[37] A. G. Voronovich. Small-Slope Approximation for electromagnetic wave scattering at a rough interface of two dielectric half-spaces. Waves in Random Media, 4:337-367, 1994.

[38] AG Voronovich and VU Zavorotny. Theoretical model for scattering of radar signals in $\mathrm{Ku}-$ and $\mathrm{C}$-bands from a rough sea surface with breaking waves. Waves in Random and Complex Media, 11(3):247-269, 2001

[39] P.A. Hwang. Observations of swell influence on ocean surface roughness. Journal of Geophysical research, 113(C12):C12024, 2008.

[40] P.A. Hwang and W.J. Plant. An analysis of the effects of swell and surface roughness spectra on microwave backscatter from the ocean. Journal of Geophysical Research, 115(C4):C04014, 2010.

[41] W.J. Plant. Whitecaps in deep water. Geophysical Research Letters, 39(16):L16601, 2012

[42] T.F. Coleman and Y. Li. An interior trust region approach for nonlinear minimization subject to bounds. SIAM J. OPT., 1996.

\section{ACKNOWLEDGMENTS}

This work was supported by the ANR project no. ANR-09BLAN-0232-01 SIMODE. A. Bringer has been funded by the Direction Générale de l'Armement. We thank Bill Plant for useful remarks and his support on the Three-scale model. 